\title{
Substance Use Class
}

National Cancer Institute

\section{Source}

National Cancer Institute. Substance Use Class. NCI Thesaurus. Code C83413.

The variable assigned to a substance usage class. 